\title{
Risk assessment of COVID-19 pandemic using deep learning model for J\&K in India: a district level analysis
}

\author{
Manzoor A. Wani ${ }^{1} \mathbb{D} \cdot$ Junaid Farooq $^{2} \cdot$ Danish Mushtaq Wani $^{3}$
}

Received: 27 July 2021 / Accepted: 11 October 2021 / Published online: 23 October 2021

(c) The Author(s), under exclusive licence to Springer-Verlag GmbH Germany, part of Springer Nature 2021

\begin{abstract}
The coronavirus disease 2019 (COVID-19) is an ongoing pandemic with high morbidity and mortality rates. Current epidemiological studies urge the need of implementing sophisticated methods to appraise the evolution of COVID-19. In this study, we analysed the data for 228 days (1 May to 15 December 2020) of daily incidence of COVID-19 cases for a district level analysis in the region of Jammu and Kashmir in the northern Himalayan belt of India. We used a deep learning-based incremental learning technique to model the current trend of COVID-19 transmission and to predict the future trends with 60-day forecasting. The results not only indicate high rates of morbidity and mortality but also forecast high rise in the incidence of COVID-19 in different districts of the study region. We used geographic information system (GIS) for storing, analysing, and presenting the spread of COVID-19 which provides key insights in understanding, planning, and implementing mitigating measures to tackle the current spread of the pandemic and its possible future scenarios. The existing disparity in health care facilities at district level is shown in relation to the spread of disease. The study results also highlight the need to upgrade health care infrastructure in the study region to control the current and future pandemics. These results could be useful for administration and scientific community to develop efficient short-term and long-term strategies against such diseases.
\end{abstract}

Keywords COVID-19 · Deep learning · GIS · Risk assesment

\section{Introduction}

The coronavirus disease 2019 (COVID-19) pandemic has been a tale of morbidity and mortality across the globe. Ongoing pandemic has eventually urged the need of implementing sophisticated methods in epidemiological studies to appraise the current and future trends of COVID-19

Responsible Editor: Lotfi Aleya

Manzoor A. Wani

manzoorwani8559@gmail.com

Junaid Farooq

junaid_phd017@nitsri.ac.in

Danish Mushtaq Wani

wanidanishmushtaq@gmail.com

1 Department of Geography and Regional Development, University of Kashmir, 190006 Srinagar, India

2 Department of Electrical Engineering, National Institute of Technology Srinagar, 190006 Srinagar, India

3 Bharat Sanchar Nigam Limited (BSNL), Jammu and Kashmir, Sopore, India pandemic that affects human lives and results in serious financial problems due to prolonged and unavoidable lockdowns. Historical outbreaks of various infectious outbreaks offer some understanding of the spatial and temporal course of current infectious pandemic, which is useful to estimate the current and future pattern of COVID-19 pandemic (J\&K Advisory Committee Report 2021). The COVID-19 pandemic caused by severe acute respiratory syndrome coronavirus 2 (SARS-CoV-2) was initially reported in patients with comorbidity such as pneumonia in the Wuhan city of China and was supposed to spread in the regions of Southeast Asia owing to geographical proximity and substantial travel connections (Sajadi et al. 2020). However, instead of Southeast Asia, COVID-19 diffused to other parts of the globe such as Europe and North America which became the largest COVID-19 epicentres with high cases of morbidity and mortality (Sajadi et al. 2020).

Countries around the globe are striving hard to mitigate the spread of COVID-19 by formulating and executing various strategies such as countrywide lockdown, closing schools, halting transport, closing regional, national, and international borders (Subramanian et al. 2020). Developing 
and under-developed countries are at a higher risk due to poor health care infrastructure, weak socio-economic factors, lack of awareness, poverty, low standard of living, illiteracy, and poor housing environment resulting in a weaker immune system (Sahoo et al. 2020). Poor or partial implementation of social distancing and other precautionary measures recommended for COVID-19 cause a significant increase in the rate of COVID-19 spread. It is, therefore, imperative to follow all the recommendations from WHO and local health authorities along with rapid vaccination of the population (Theeb et al. 2021).

Machine Learning (ML) and Cloud Computing can be used to efficiently track the progression of this pandemic across the globe by predicting future trends of COVID-19 that could be used in designing strategies and policies to manage its diffusion (Shreshth et al. 2020). An artificial intelligence technique based on a deep convolutional neural network to detect COVID-19 patients using real-world data sets is currently used in detection of COVID-19 pandemic that is increasing exponentially with almost 35 million confirmed cases globally (Moutaz et al. 2020). Forecasting the spread of COVID-19 pandemic requires ample historical data, and it is also worth mentioning that no prediction can be fully certain (Petropoulos and Makridakis 2020). Understanding the early signs of the dynamic nature of this infectious global pandemic and appraising the overall efficacy of current measures are important in assessing its development while adopting sustainable controlled measures in the newer areas (Samuel et al. 2020).

It is important to collect, store, manipulate, analyse, and interpret COVID-19 data to disseminate necessary information and put forward rational strategies for mitigating the burden of COVID-19 pandemic (Ahmadi et al. 2020). Integration of mathematical modelling and geographical information system (GIS) can play a decisive role in mitigating the diffusion and predicting the further growth of pandemic (Ankush 2020).

Geographical knowledge about identifying the space (existence), structure, and inter-relation of the phenomena can play a crucial role in understanding and mitigating COVID-19 pandemic (ESRI 2020). Modern GIS technologies centre around web-based tools, improved data sharing, and real-time information to support critical decisionmaking (Kamel and Geraghty 2020). Dashboards exemplify those ideals and have been extremely popular in sharing and understanding the spread of SARS-CoV-2 coronavirus. Communication through map-based dashboards offers accessible information to people around the world eager to protect themselves and their communities. This improves data transparency and helps authorities disseminate information (Kamel and Geraghty 2020).

Spatial analysis of data through GIS has become an essential technique in academic schools of public health, environmental health, and in public health agencies that are responsible for disease surveillance, control, and health planning (Mayer 1983). In 2003, the international organization of health in Europe projected the benefit of using geographic information system (GIS) for the identification of diseases and understanding the features such as spatial variation of diseases, and their association to environmental factors and the health care system (Aghajani et al. 2017). With the advancement of analytical and computing power, GIS technology has boosted the importance of mapping epidemiological diseases considerably (Malczewski 2004). The mapping of disease helps in interpreting the delineated area more effectively and efficiently, which is a great asset for epidemiological planning (Anna and Daniel 2014).

In the neighbouring country of Pakistan which shares geographical, cultural, ethnic, and economic status with India, Saddique et al. (2021) and Adnan et al. (2021) have studied various aspects of COVID-19 spread. Abdelhafez et al. (2021) recently studied the effect of weather data on the spread of COVID-19. Anser et al. (2021) studied the impact of coal combustion, nitrous oxide emissions, and traffic emissions on COVID-19 cases. Qaid et al. (2021) studied the long-term statistical assessment of meteorological indicators and COVID-19 outbreak in hot and arid climate. Sahoo (2021) studied the significance between air pollutants, meteorological factors, and COVID-19 infections.

The need of the hour is to get location-wise risk assessment so that timely preventive measures can be taken at the local level. Countries across the globe in cooperation with WHO are engaged in ensuring not only the development of effective vaccines but their availability to all people as well. Thus far, WHO has effectively rolled 7 different vaccines across three formats to boost body's natural immune system (WHO 2021).

India which is the second largest country in population and 7th largest in area was hit by COVID-19 pandemic in early 2020 with prolonged continuation and meagre declined trend in the winter months. Till now, the country as a whole has marked the second highest number of infected cases with nearly 2.83 crore infected cases reported and over 3.35 lac deaths (https://github.com/CSSEGISandData). The union territory of Jammu and Kashmir following the national trend has also shown a high rise in daily cases and is expected to increase manifold in the coming months, keeping in view the nature of perpetual backwardness in terms of socioeconomic factors, political instability, unique topographical identity, prevalent respiratory problems, and current available health care infrastructure (Wani et al. 2020) might induce a new pandemic epicentre in Jammu and Kashmir. In this study, we analysed the data for 228 days (1 May to 15 December 2020) of daily incidence of COVID-19 cases for a district level analysis in Jammu and Kashmir. We used a deep learning-based incremental learning technique to 
model the current trend of COVID-19 transmission and to predict the future trends with 60-day forecasting. We used geographic information system (GIS) for storing, analysing, and presenting the spread of COVID-19.

\section{Methods}

\section{Study area}

The present study was carried out in the northwestern region of the Himalayas surrounded by snow clad mountains, carpeted with lush green meadows, undulating topography, distinctive climate region, varied geo-physical environment, and unique social cultural setup. Total human population of 12.27 million is residing in an area of $42,241 \mathrm{~km}^{2}$. The area has two administrative regions, i.e., Jammu region covering $62 \%$ of the total area and Kashmir region with $38 \%$ of the total area. Both regions contribute almost equal share of population with high population density (432 per square kilometre) in the Kashmir valley region. The region has been demarcated into 20 districts with 10 from each region for administrative purposes. The variation in the topography and climate has not only affected the socio-cultural and economic conditions of the people but plays a potent role in the overall health scenario (Wani et al. 2019). The geographical identity of the area with discrete physical, socio-economic, and behavioural environment imposes a great challenge to overall respiratory health (Wani et al. 2020). Figure 1 shows the map of the study area.

\section{COVID-19 data}

Data of daily incidence cases of COVID-19 in the study region from 1 May 2020 to 15 December 2020 were obtained from the continuously updated Twitter handle of the Department of Information and Public Relations, Govt. of Jammu Kashmir (DIPR) (https://twitter.com/diprjk).

\section{Mathematical modelling}

Predicting future and current trends in the epidemiological studies is an important problem for public health and has gained an increasing attention in the data mining and machine learning communities (Yuexin et al. 2018). A modified model of Susceptible Infected Recovered (SIR), known as SIRD (Susceptible-Infected-Recovered-Deceased) Model, was used in this paper to simulate the transmission dynamic behaviour of COVID-19 infection rates as described and validated by Farooq and Bazaz (2020a). This model is fatal unlike a typical non-lethal SIR model which means that there is a positive probability of an infected person succumbing to the disease. A typical SIR model assumes that the recovered group of patients gains full immunity from reinfection. However, this model accommodates the possibility of a recovered person being reinfected. The impact of new births and unrelated deaths is ignored, and the total population is assumed to remain constant with random distribution. The problem of parameter estimation or learning for this model was solved by using a deep learning technique known as incremental learning as described and implemented by Farooq and Bazaz (2020b). Training data is needed before executing a learning algorithm or estimation technique. However, in an epidemic like situation, the training data becomes available only with the progress of disease with time. Thus, the model must train and execute simultaneously while the model parameters keep varying with time. Further, the training data gets accumulated over time increasing the computational burden. Therefore, deep learning was used to train the model in an adaptive fashion such that the need to keep rebuilding the model whenever new training data sets are received is eliminated.

This model is intelligent, non-intrusive, adaptive, realtime, and online in nature; therefore, it can be used for monitoring, forecasting, and simulation of transmission dynamics and growth of any epidemic without any loss of efficiency, accuracy, fidelity, or computational performance which generally arise due to long run time, large size of training data, increasing computational complexity, change in virus behaviour due to mutations, change in government policies, etc. Even if the epidemic continues for decades in the whole world, the model will keep running accurately on a daily basis without loss of performance.

In the study area, we run a deep learning model to daily recorded COVID-19 cases, i.e., prevalence, recovery, and death rates, from 1 May to 15 December 2020, with 60-day forecasting in all district of the study area.

We have considered the training data for 228 days and prediction of 60 days. Thus, our prediction horizon is approximately one-fourth $(26.31 \%)$ of the data horizon which seems to be a reasonable choice as the prediction error increases with an increasing prediction horizon.

\section{Statistical techniques}

Kendal's rankings were assigned to the COVID-19 cases (prevalence, recovery, and death rate), and the composite index method was adopted to measure the intensity of COVID-19 cases (prevalence, recovery, and death rate) district-wise to generate a heat index map using GIS techniques. Determining the district-wise disease intensity of COVID-19 incidence cases (prevalence, recovery, and death rates) is an essential tool for understanding the spatial pattern and intensity of COVID-19 pandemic in any area as it clearly elucidates the relative dominance of a particular disease in any area (Wani et al. 2019). In this 

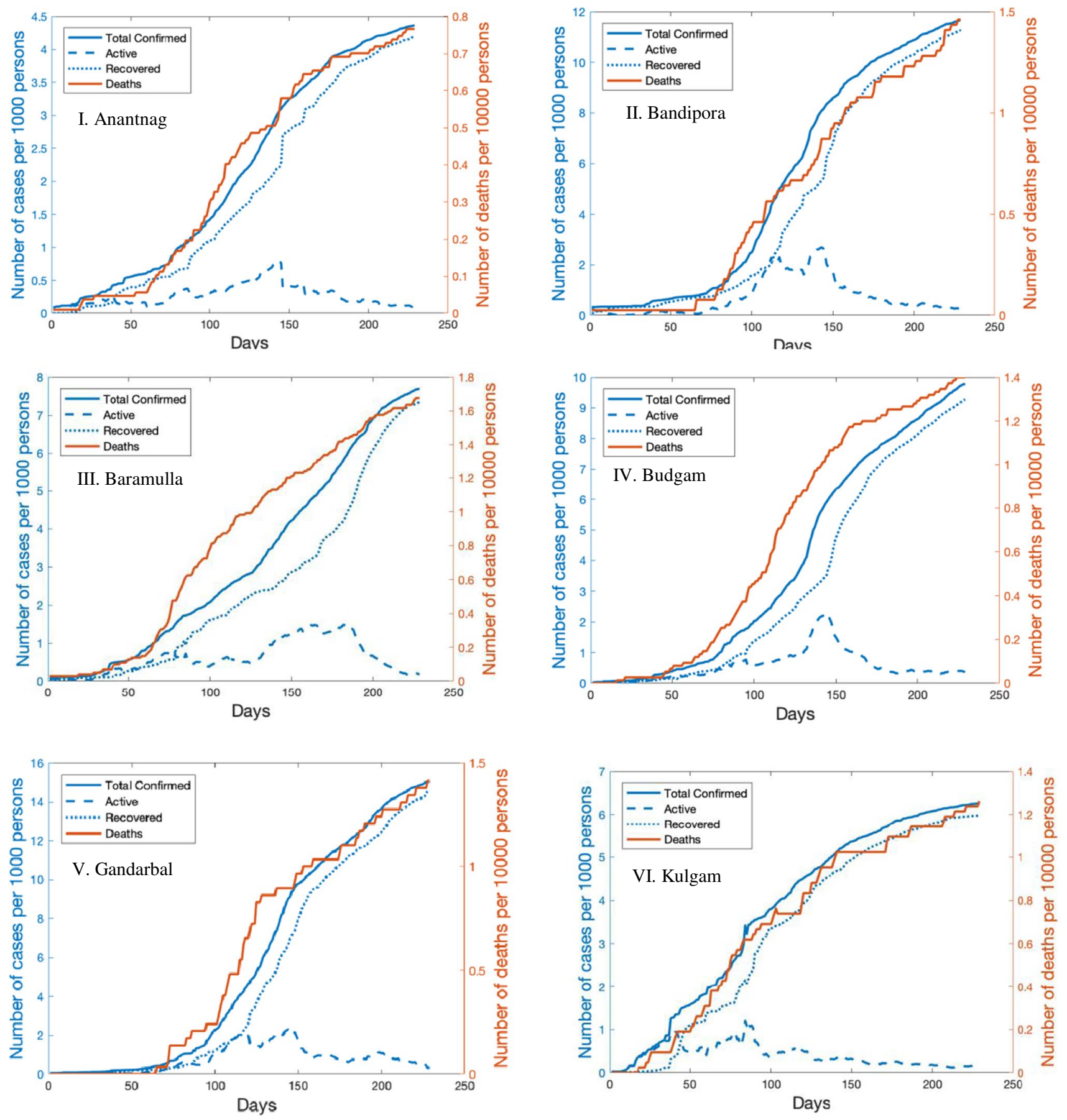

Fig. 1 (I-X): COVID- 19 casses (active, recovery, deaths) in the districts of Kashmir dvision from 1 May to 15 December 2020

study, we used Kendal ranking method by assigning ranks to the COVID-19 cases (incidence, recovery, and death rates). To show the spatial pattern of disease intensity, we used a composite index technique on the obtained rank scores of each COVID-19 case (incidence, recovery, and death rates) of each district as
Composite index $=C I=\sum_{j=1}^{n} \frac{X_{i j}}{X_{j}}$

Here, $X i j=$ value of $j$ th variate for $i$ th district; $n=$ number of variables. 

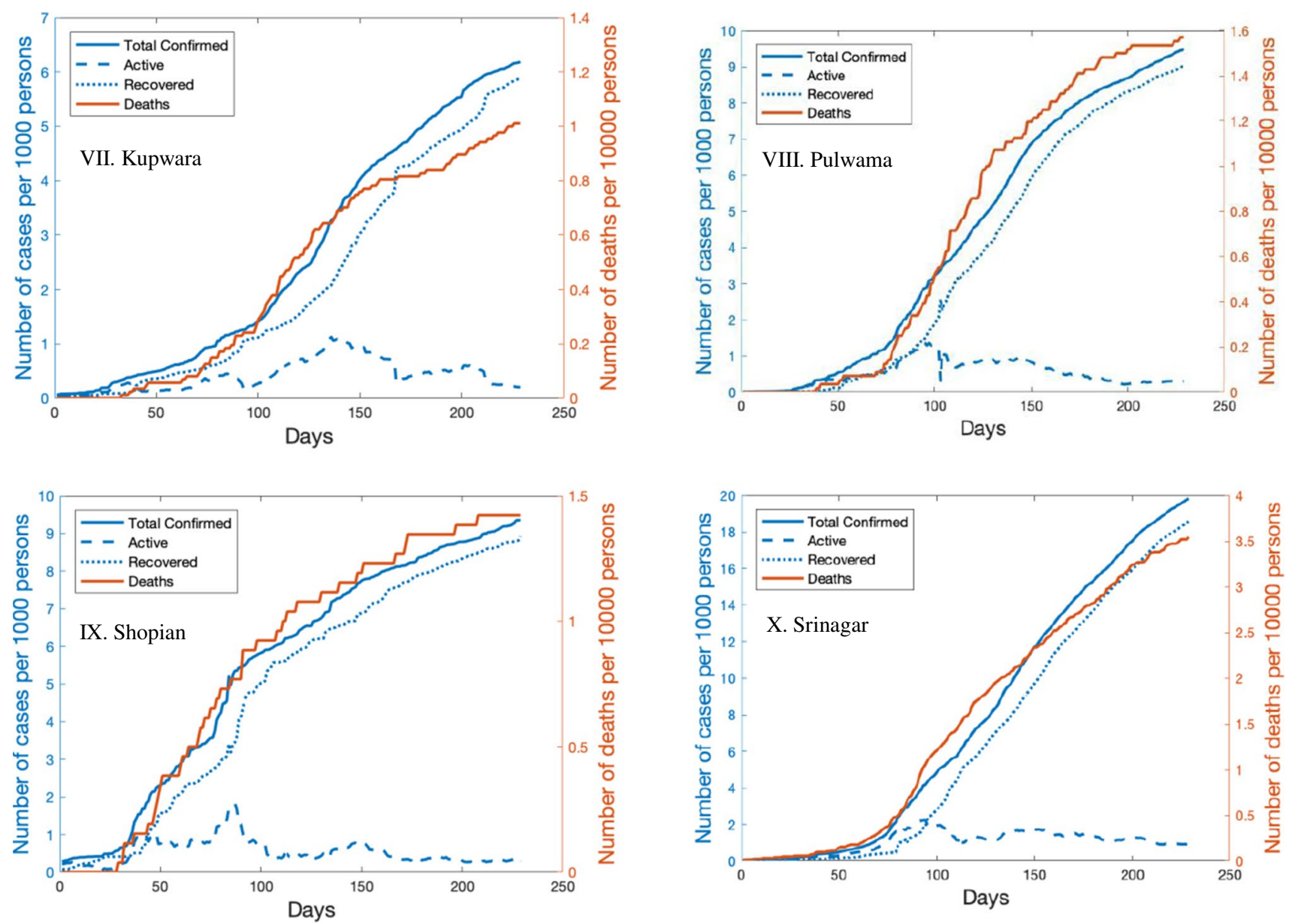

Fig. 1 (continued)

\section{Demographic data}

District-wise demographic data, i.e., total population, growth rate, density, literacy rate, were obtained from the current available source, i.e., JK Statistical Digest 2016 and Census of India 2011.

\section{Health care infrastructure}

It is important to appraise the health care infrastructure in the current scenario of COVID-19 pandemic as it raises several concerns about the capacity of the government to tackle the existing health care facilities (Atiqa and Sana 2020). In this connection, an evaluation in the overall health care infrastructure was assessed to estimate the prepared measures to mitigate the incidence of COVID-19 pandemic at the inter-district level. The details on quantification of health care infrastructure are given in the Supplementary Information.

\section{Mapping}

Study area and heat maps related to the incidence of COVID-19 cases (active, recovery, and death rate) in the area were stored, manipulated, analysed, and presented in Arc GIS 10.2 software.

\section{Results and discussion}

\section{Evolution of COVID-19 cases}

Diffusion of COVID-19 across the globe has taken a huge toll on human lives. This study pertains to appraise interdistrict variation in the pattern of COVID-19 cases (active, recovery, and death rate). As seen in Figs. 1 and 2, there is a sharp rise in the incidence of COVID-19 active cases and death rates from the $60^{\text {th }}$ day of data observation in the whole area. During this period (July-August), the whole region experienced a sharp rise in the air temperature and relative humidity which nullifies the role of meteorological 

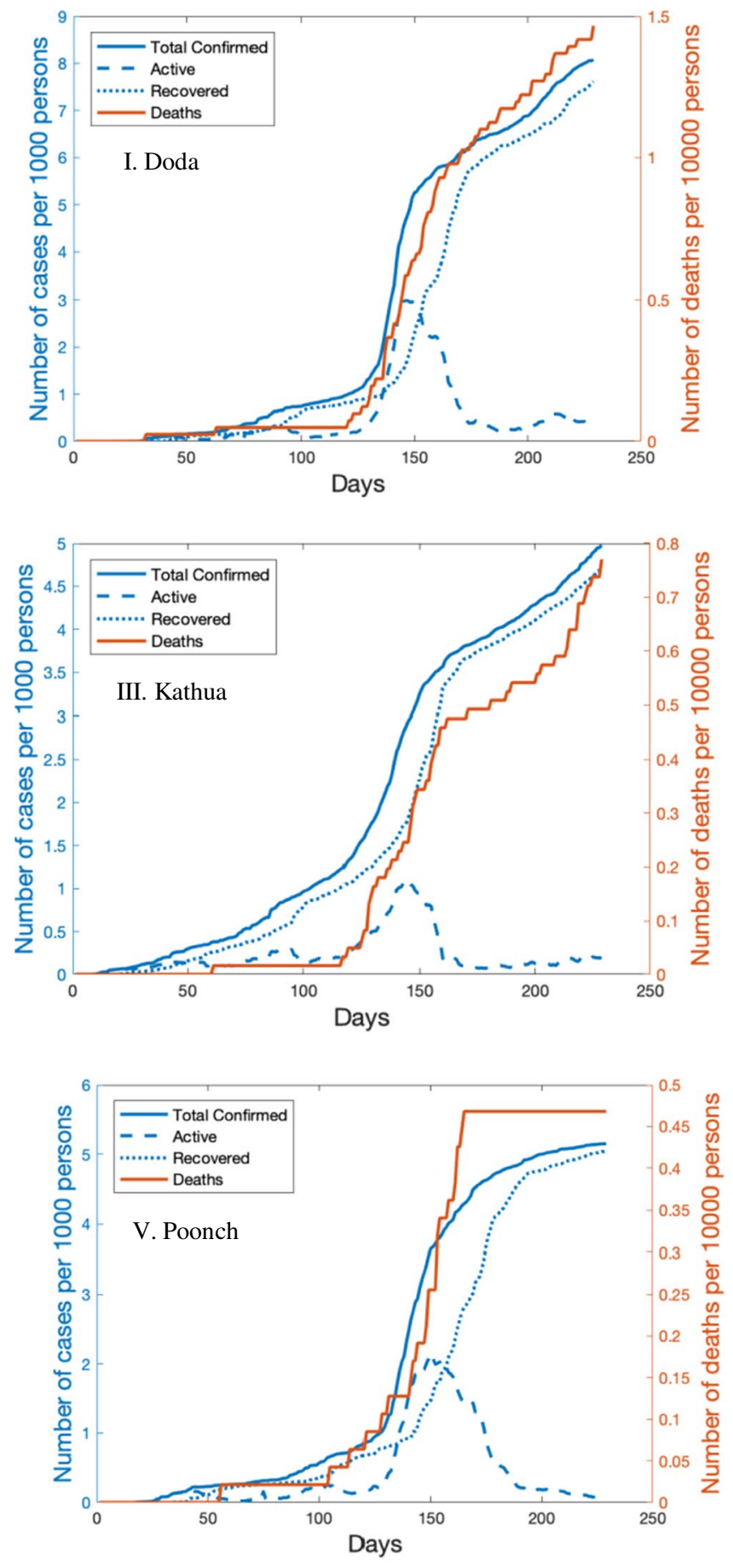
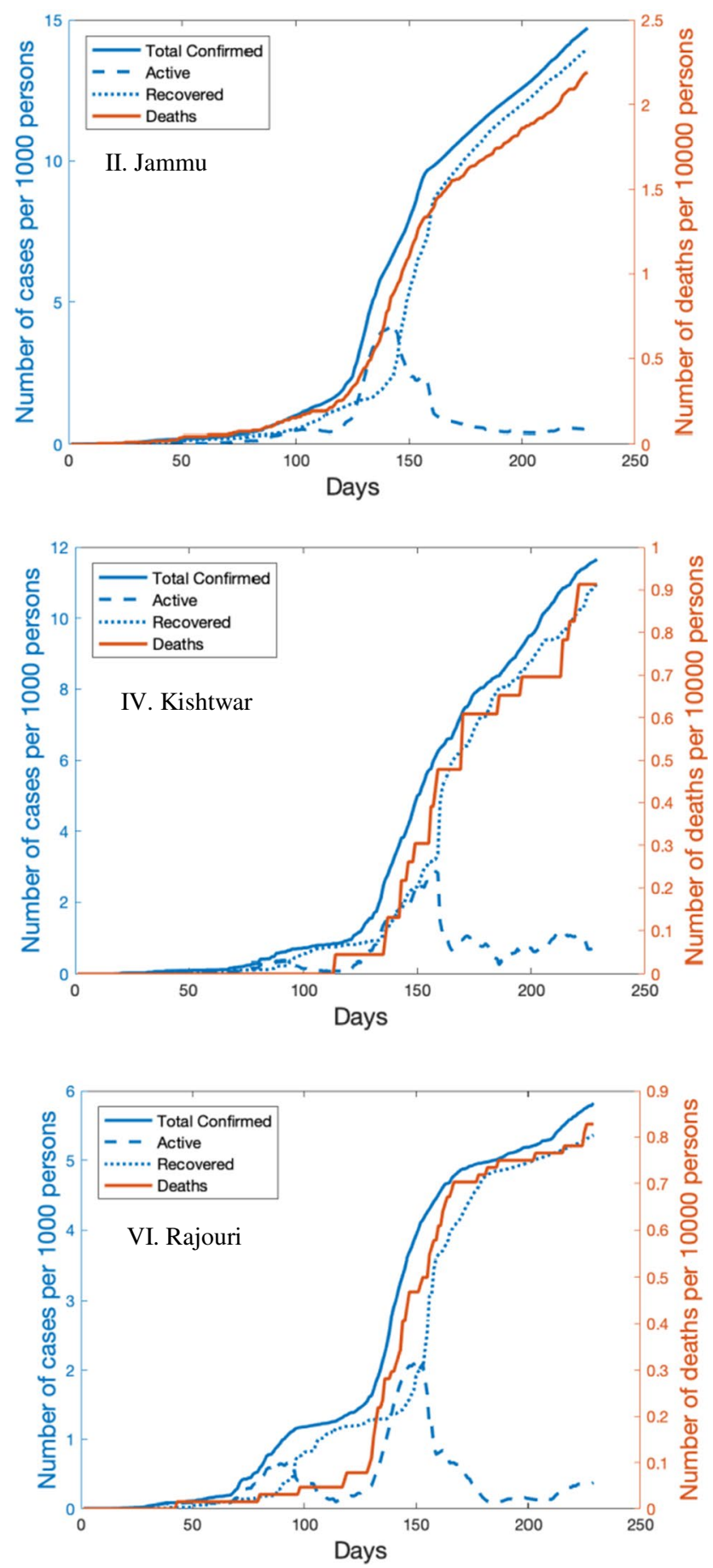

Fig. 2 (I-X): COVID-19 cases (active, recovery, deaths) in the districts of Jammu dvision from 1 May to 15 December 2020

parameters on the incidence of COVID-19 in the region (Wani et al. 2020). In the study, we found spatial temporal variation in the incidence of COVID-19 cases with high infectious and death rates in the districts of Kashmir division as shown in Fig. 1 (I-X). In comparison, districts of Jammu division, Fig. 2 (I-X), show low rates of infection, death rates, and high recovery rate during the given time period.
Albeit it is still preliminary to relate any major etiological cause in the spatial variation with diffusion of COVID-19 cases in these two different geographical proximities, the variation in the incidence cases can be asserted with the variation in the geographical identity of these two regions which directly affects their modes of life (Wani et al. 2020). Figure 3 shows the total COVID-19 cases with prediction 

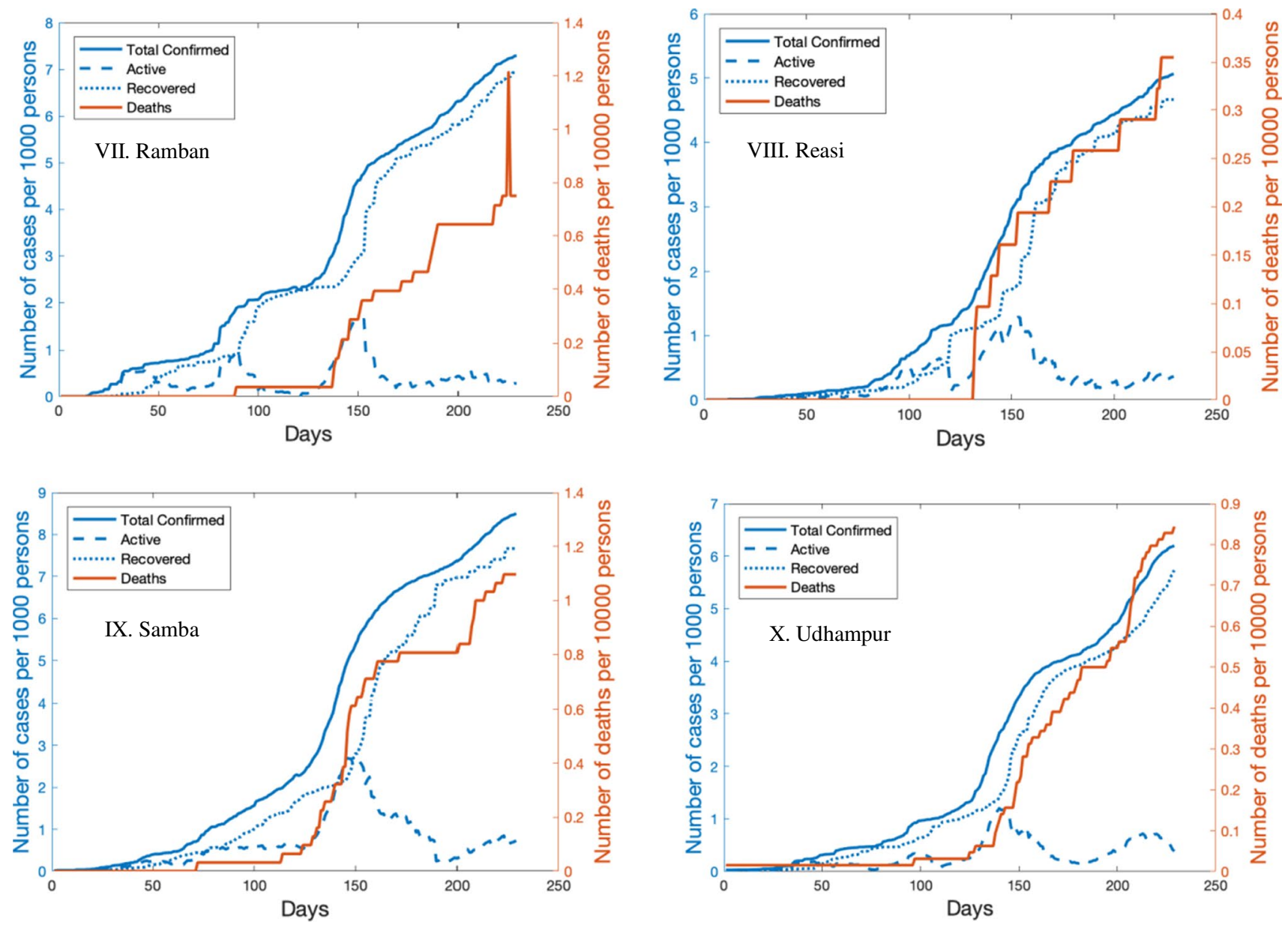

Fig. 2 (continued)

and model validation for the whole region. In Fig. 3, it is clearly seen that the area is witnessing a sharp rise in COVID-19 cases (active and death rates) with more people getting infected strengthening the infection chain and adding burden to limited health care resources. The deep learning model shows high data accuracy with least mean absolute error $(\mathrm{MAE}=1.1 \%)$. Kashmir region is known for its personified natural landscape and soothing climate and, therefore, witnesses high tourist flow throughout the year. This could be the main reason for high COVID-19 diffusion as many cases were linked to the UK variant of the virus as compared to Jammu where virus of Indian variant was found more dominant (J\&K Advisory Committee Report 2021). Besides the variant nature of virus, spatial variation is seen in the socio-economic status as shown in Table $\mathrm{S} 1$ which also affects the COVID-19 incidence such as high population, high population density, low literacy rate, political instability, and other related socio-economic problems, which are more common in the valley part (Kashmir) of region. With unique geographical identity, people of the valley region are more prone to respiratory ailments, cardiovascular diseases, hypertension, diabetes, etc. (Wani et al. 2019). Heat index maps showing the intensity of COVID-19 cases (active, recovery, and death rates) for each district are shown in Fig. 4a-c. Spatial variation in the incidence of COVID19 cases is observed as the districts with high cases from urban areas and districts with high population concentration such Srinagar, Jammu, Budgam, and Baramulla show high positivity and fatality rates. These hotspot areas need quick response in providing and managing COVID-19 health care facilities to tackle COVID-19 surges by strengthening the preparedness and mitigation measures. The heat index maps generated are helpful in identifying hotspot areas and in proper response management in resource-allocation measures for COVID-19 during recovery phases. District-wise analysis of COVID-19 is shown in detail in the Supplementary Information. The results suggest that the pandemic will continue to grow in all districts of Jammu and Kashmir in the near future. Therefore, the administration should enhance their strategies and upgrade the health infrastructure in each district to mitigate the effect of the pandemic. 

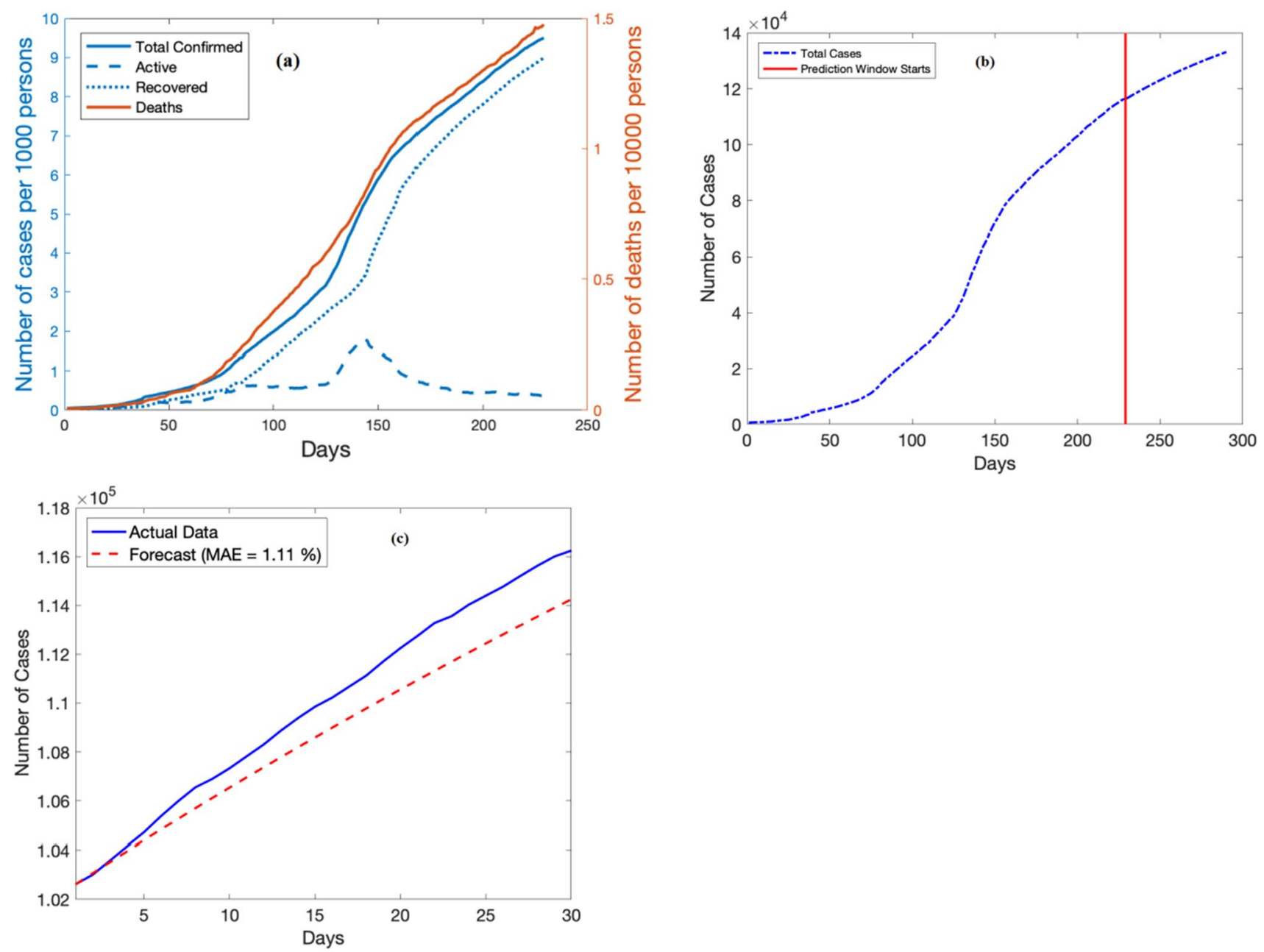

Fig. 3 Total COVID-19 cases in Jammu and Kashmir (1 May to 15 December 2020): (a) disease evolution, (b) prediction, (c) model validation and MAE for past 1 month

\section{COVID-19 health care facilities}

COVID-19 pandemic has possessed serious challenges to the current health care infrastructure around the world. In this study, we appraised the current scenario of COVID-19 health care amenities available at inter-district level. Albeit administration has stepped towards the progression of health care infrastructure, but it seems virtually impossible for the administration in Jammu and Kashmir to improve the objectives of various health and welfare program as propounded by WHO. As shown in Table SII, high inter-district disparities are seen in the current health care infrastructure such as isolation beds with and without ICU beds, availability of oxygen-supported beds for confirmed and suspected cases, and ventilators to cope with COVID-19 severity. Scarcities in COVID-19 health care facilities were seen more in the districts of Kashmir division with continuous spike in the COVID-19 cases in the region. High patient ratio with available COVID-19 health facilities was seen in the entire region. Poor and insufficient health care facilities available in the periphery districts of the region are also possessing challenges to the nodal health care institutions, resulting in immense burden while tackling COVID-19 infectious cases. The extent of inter-regional disparity of patient ratio and COVID-19 health facilities can be analysed by the looming scenario of the current health care system available in the urban regions of the study area, i.e., Srinagar sharing (1:6) and Jammu (1:3) isolation beds without ICU for confirmed cases. Worse situation was observed in other districts of Kashmir division such as Bandipora (1:10), Ganderbal (1:5), and Jammu (1:5). Patient ratio increases with critical COVID-19 health care facilities in the entire districts of the study region, i.e., high patient ratio seen in oxygensupported beds in the districts of Kathua (1:119), Srinagar (1:87), Rajouri (1:45), and Budgam (1:36). Sharing of lifesaving ventilators shows an increasing burden, i.e., 1:405 in the entire region indicating that 405 COVID-19 patients can avail one ventilator at a particular facility if the severity rate 


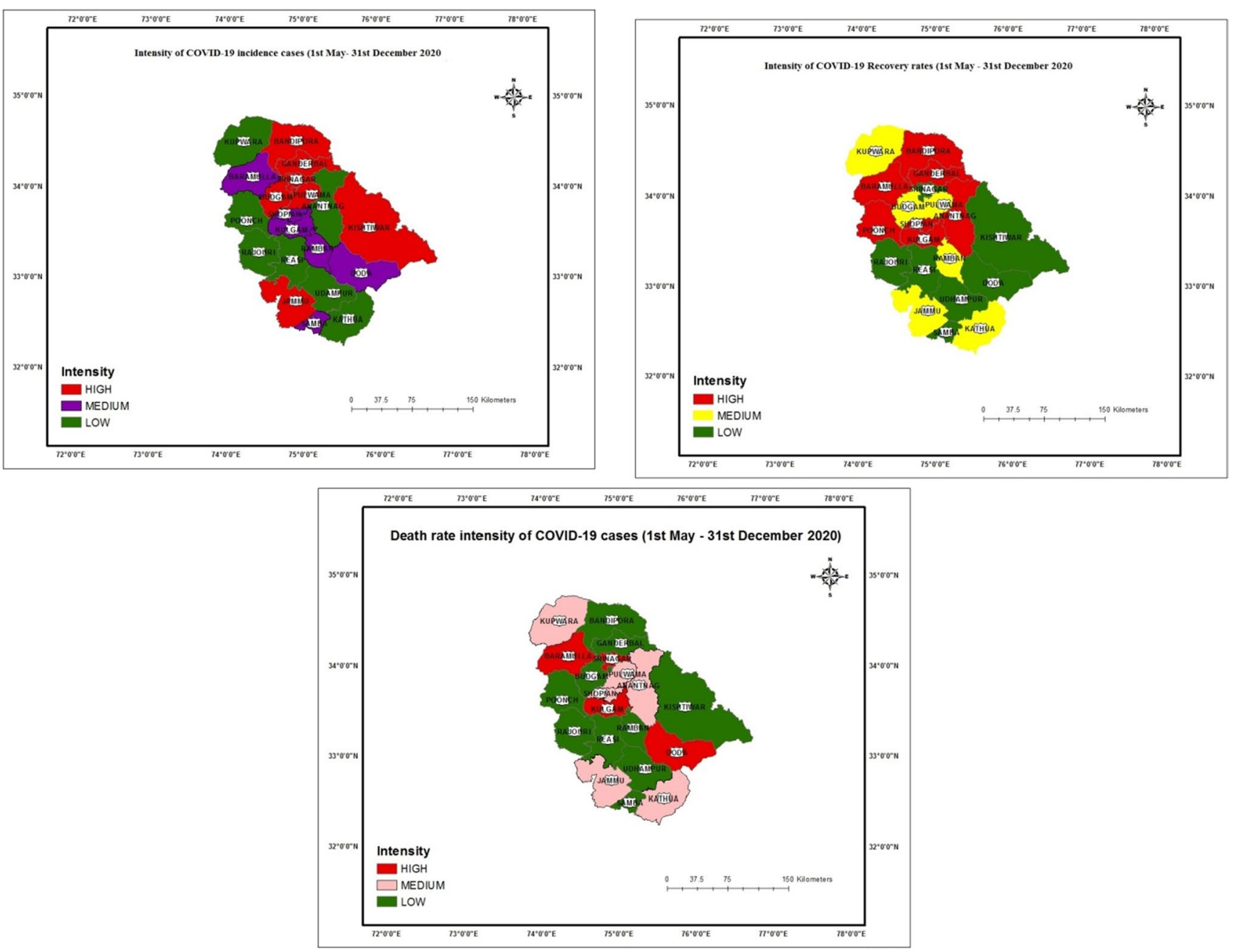

Fig. 4 Intensity of COVID-19 cases (incidence, recovery, and death rates) from 1 May to 31 December 2020

increases; this patient ratio is seen high in Srinagar (1:1546), Pulwama (1:1322), and Kulgam (1:962) districts. It is clear from the preceding discussion that the whole region lacks basic COVID-19 health facilities, with extreme regional disparities prevailing in the region which adds more hurdles while mitigating this pandemic. With the current health care infrastructure available, the study area as a whole is highly vulnerable to the variant nature of coronavirus without proper and adequate mitigating measures.

India has the second largest population in the world; therefore, the progress of COVID-19 in India affects the evolution of the disease globally. This paper presents the first district level study of COVID-19 evolution and prediction in India. Moreover, the study relates the COVID19 progression with existing health infrastructure and suggests that disparity in health facilities initially endangers the disadvantaged sections of the society, but ultimately affects all sections. For example, urban centres are generally considered safer than rural areas due to better health facilities; however, in case of an epidemic, the rural population seeks treatment from urban centres and results in the spread of the disease everywhere. Same is the case with economic disparity based on social factors. Thus, this study presents a case for equitable distribution of resources in all sections of the society for the benefit of everyone.

The results of this study could be useful for the administration and policy makers to manage and control the pandemic. It also provides important insights regarding the need to upgrade the health infrastructure in Jammu and Kashmir to combat different diseases effectively in the future. The effect of climatic conditions, political scenario unique to the region, the effect of migration in population, and geographical differences between regions have not been considered. These factors are interesting for analysis in future research. 


\section{Conclusion}

The threat posed by the current COVID-19 pandemic in India seems to get accelerated in the coming months as seen in the variant behaviour of SARS-CoV-2. It is expected that the world might experience multiple waves of COVID-19 pandemic, each with increased intensity. Keeping in view the dynamic nature of coronavirus, it is expected that positivity and fatality rate will surge. A little pause of dormancy is actually dimming the responsive behaviour resulting in loosening in the protective measures such as social distancing and other SOP guidelines recommended for COVID-19. This might prove more fatal than the previous pandemic waves as seen in the variant nature of coronavirus that is gaining momentum and resistance with each new variant, and presently the threat of new delta variant is looming across the globe. The purpose of the present study was to estimate the current and future risk involved due to COVID19 using two sophisticated and advanced tools, i.e., deep learning modelling and GIS to understand and respond to the ongoing pandemic more effectively and efficiently. In the study area, high cases of COVID-19 were seen in many districts and are expected to increase manifold in the coming months with each escalation in COVID-19 wave. Insufficient health care infrastructure, high population, illiteracy and lack of perception, misconceptions, and lack of trust in the current vaccination drive make it a huge concern for the administration and policy makers. It is noteworthy that the country (India) has developed two vaccines (COVISHIELD and COVAXIN) and has initiated mass vaccination drive across the country in various phases because of huge population. The vaccination drive faces unprecedented constraints due to ambiguity regarding the clinical validation of the rolled out COVID-19 vaccines, large population, lack of scientific perception, and traditional bound beliefs.

It is important and utmost priority to follow a cost-free approach to cope with COVID-19 pandemic, i.e., follow the guidelines and policies set by the World Health Organization (WHO) in general and local administration, i.e., maintaining physical distance, proper hygiene, use of masks to avoid the further transmission. It is also mandatory that government administration must take urgent initiatives to upgrade health care infrastructure especially in the hotspot districts to mitigate the burden of the COVID-19 pandemic in the future.

Supplementary Information The online version contains supplementary material available at https://doi.org/10.1007/s11356-021-17046-9.

Acknowledgements Authors are thankful and offer sincere gratitude to the Department of Information and Public Relations, Govt. of Jammu Kashmir (DIPR) for maintaining, updating, and publishing COVID-19 data on daily basis. The authors are also thankful to the Census Department of India and Directorate of Statistics and Economics, Jammu and Kashmir for maintaining and providing demographic data. We would also like to thank Dr. Muhammad A. Bazaz (Associate Professor) and Head of the Department of Electric Engineering-National Institute Srinagar, India for helping and supervising us during this work.

Author contribution MAW and DMW have designed the work. MAW, JF, and DMW collected the required data. Data analysis and interpretation are done by MAW, JF, and MAB. MAW, JF, and DMW drafted the manuscript.

Data availability COVID-19 data are provided by the Department of Information and Public Relations, Govt. of Jammu Kashmir (DIPR). Data link: DIPR-J\&K (@diprjk). Twitter: https://twitter.com/diprjk. Demographics data can be downloaded from www.censusindia.gov. in www.ecostatjk.nic.in.

\section{Declarations}

Ethics approval and consent to participate Not applicable.

Consent for publication Not applicable.

Competing interests The authors declare no competing interests.

\section{References}

Abdelhafez E, Dabbour L, Hamdan M (2021) The effect of weather data on the spread of COVID-19 in Jordan. Environ Sci Pollut Res 28:40416-40423. https://doi.org/10.1007/s11356-020-12338-y

Adnan S, Hanif M, Khan AH, Latif M, Ullah K, Bashir F et al (2021) Impact of heat index and ultraviolet index on COVID-19 in major cities of Pakistan. J Occup Environ Med 63(2):98

Aghajani J, Farnia P, Velayati AA (2017) Impact of geographical information system on public health sciences. Biomed Biotechnol Res J 1(2):94-100

Ahmadi K, Dashti MF, Delgosha MS. (2020) Geographical distribution of COVID-19 in the World and Iran; investigation of possible roots. J Family Med Primary Care; 9(8). Doi:https://doi.org/10. 4103/jfmpc_733_20

Ankush K (2020) Modeling geographical spread of COVID-19 in India using network based approach. medRxiv preprint. https://doi.org/ 10.1101/2020.04.23.20076489

Anna B, Daniel D (2014) Mapping disease patterns. Statistics reference online, Wiley StatsRef. https://doi.org/10.1002/9781118445112. stat06102.pub2

Anser K, Godil DI, Khan MA et al (2021) The impact of coal combustion, nitrous oxide emissions, and traffic emissions on COVID19 cases: a Markov-switching approach. Environ Sci Pollut Res. https://doi.org/10.1007/s11356-021-15494-x

Atiqa K, Sana A (2020) COVID-19 challenges for healthcare system in Pakistan. Asian Bioethics review. https://doi.org/10.1007/ s41649-020-00139-x

CSSEGIS and COVID-19 (2021) COVID-19 data repository by the center for systems science and engineering (CSSE) at Johns Hopkins University; https://github.com/CSSEGISandData

ESRI (2020) Geographic information system for corona virus planning and response. Esri.com/covid-19. Accessed 7 Jun 2021

Farooq J, Bazaz MA (2020a) A deep learning algorithm for modeling and forecasting of covid-19 in five worst affected states of India. Alexandria Eng J. https://doi.org/10.1016/j.aej.2020.09.037

Farooq J, Bazaz MA (2020b) A novel adaptive deep learning model of covid-19 with focus on mortality reduction strategies. Chaos, 
Solitons \& Fractals 138:110148. https://doi.org/10.1016/j.chaos. 2020.110148

J\&K Advisory Committee Report (2021) Action plan for COVID-19 third wave, J\&K (UT)

Kamel B, Geraghty EM. (2020). Geographical tracking and mapping of coronavirus disease COVID-19/severe acute respiratory syndrome coronavirus 2 (SARS-CoV-2) epidemic and associated events around the world: how 21 st century GIS technologies are supporting the global fight against outbreaks and epidemics. Int J Health Geographics; 19(8). https://doi.org/10.1186/s12942-020-00202-8.

Malczewski J (2004) GIS, spatial epidemiology and health care planning. Geography and health: a study in medical geography. APH publishing Corporation, New Delhi

Mayer JD (1983) The role of spatial analysis and geographic data in the detection of disease causation. Social Sci Med 17(6):1213-1231

Moutaz A, Albara A, Abdelwadood M, Ajit A, Vansh J, Salah A (2020) COVID-19 prediction using deep learning. International Journal of Computer Information Systems and Industrial Management Applications. ISSN 2150-7988 12(2020):168-181

Petropoulos F, Makridakis S. (2020) Forecasting the novel coronavirus COVID-19. Plos One 15(3). https://doi.org/10.1371/journal.pone. 0231236

Qaid A, Bashir MF, Remaz-Ossen D et al (2021) Long-term statistical assessment of meteorological indicators and COVID-19 outbreak in hot and arid climate, Bahrain. Environ Sci Pollut Res. https:// doi.org/10.1007/s11356-021-15433-w

Saddique A, Adnan S, Bokhari H, Azam A, Rana MS, Khan MM, Hanif M, Sharif S. (2021) Prevalence and associated risk factor of COVID-19 and impacts of meteorological and social variables on its propagation in Punjab, Pakistan. Earth Syst Environ 1-14. https://doi.org/10.1007/s41748-021-00218-5

Sahoo H, Mandal C, Mishra S, Banerjee S. (2020) Burden of COVID19 pandemic: perspectives from health care infrastructure. International Institute for Population Sciences, Mumbai, 24

Sahoo MM (2021) Significance between air pollutants, meteorological factors, and COVID-19 infections: probable evidences in India. Environ Sci Pollut Res 28:40474-40495. https://doi.org/10.1007/ s11356-021-12709-Z

Sajadi MM, Habibzadeh P, Vintzileos A, Shokouhi S, Miralles F, Amoroso A (2020) Temperature, humidity and latitude analysis to estimate potential spread and seasonality of coronavirus diseases 2019 (COVID-19). JAMA NetworkOpen. https://doi.org/10.1001/ jamanetworkopen.2020.11834
Samuel O S, Abdul A I, Daouda S, Ismaila O I, Francis IJ (2020) Understanding the early evolution of COVID-19 disease spread using mathematical model and machine learning approaches. Global J Sci Front Res XX (V)

Shreshth T, Shikhar T, Rakesh T, Sukhpal SG (2020) Predicting and growth trend of COVID-19 pandemic using machine learning and cloud computing. MedRxiv, preprint https://doi.org/10.1101/2020. 05.06.20091900

Subramanian SV, Omar K, Weixing Z, Rockli K. (2020) Geo-mapping of COVID-19 risk correlates across districts and parliamentary constituencies in India. Harvard data science Review. https://creat ivecommons.org/license/by/4.0/legalcode

Theeb AA, Abdullah A, Reem AA, Faten AK, Hisham HA, Abdullah MA, Mohammad A, Eman MA, May BJ, Mohamed MA, Anwar AJ (2021) The current reproduction number of COVID-19 in Saudi Arabia: is the disease controlled? Environ Sci Pollution Res. https://doi.org/10.1007/s11356-021-14217-6

Wani MA, Kawoosa W, Mayer IA (2019) Mapping of morbidity pattern respiratory diseases: medical block analysis in the northern belt of India. Geo J Springer 86:455-474. https://doi.org/10.1007/ s10708-019-10065-7

Wani MA, Wani DM, Naik S, Mayer IA (2020) Geographical vulnerability to respiratory infections using GIS technique-micro analysis study in the Himalayan region of India. Geo J 85(5). https:// doi.org/10.1007/s10708-020-10307-z(0123456789().,-volV() 0123456789

WHO (2021) Coronavirus disease (COVID-19)/COVID-19 vaccines https://www.who.int/emergencies/diseases/novel-coronavirus2019/covid-19-vaccines. Accessed 15 Jun 2021

Yuexin W, Yiming Y, Hiroshi N, Masaya S. (2018) Deep learning for epidemiological predictions. The 41st International ACM SIGIR conference on research and development in information retrieval, July 8-12, Ann Arbor, MI, USA, https://doi.org/10.1145/32099 78.3210077

Publisher's note Springer Nature remains neutral with regard to jurisdictional claims in published maps and institutional affiliations. 